\title{
Double Differential Neutron Yields From Thick Targets Induced By Relativistic Carbon And Uranium Beams
}

\author{
K. Gunzert-Marx ${ }^{1,2}$, T. Radon ${ }^{1}$, G. Fehrenbacher ${ }^{1}$, F. Gutermuth ${ }^{1}$, D. Schardt ${ }^{1}$ \\ ${ }^{1}$ Gesellschaft für Schwerionenforschung Planckstraße 1, 64291 Darmstadt, Germany \\ ${ }^{2}$ Siemens Medical Solutions, Allee am Röthelheimpark 2, 91052 Erlangen, Germany \\ E-mail: t.radon@gsi.de
}

In order to design appropriate shielding for the next generation high-intensity acceleratorfacilities like e.g. FAIR, knowledge of neutron emissions induced by relativistic heavy ion beams stopping in accelerator components or concrete shielding is indispensable. Measured neutron spectra can directly be used as source terms for Monte-Carlo transport calculations.

Thus measurements were carried out in Cave B of the SIS accelerator at GSI in order to determine double differential yields of fast neutrons produced by heavy ion beams in thick stopping targets. Three different beam-target combinations were investigated: (a) 12C beam (400 MeV/u) stopping in a $20 \mathrm{~cm}$ thick graphite target, (b) $12 \mathrm{C}$ beam ( $1 \mathrm{GeV} / \mathrm{u})$ stopping in a 20 $\mathrm{cm}$ thick iron target, and (c) $238 \mathrm{U}$ beam $(1 \mathrm{GeV} / \mathrm{u})$ stopping in a $20 \mathrm{~cm}$ thick iron target. Fast neutrons emerging from the targets were detected by a BaF2 scintillator positioned at various angles with respect to the primary beam axis. The neutron energy was obtained from time-offlight measurements.

A comparison of the measured double differential yields with the Monte-Carlo code FLUKA and measurements with the so called Large Area Neutron Detector (LAND) at GSI are presented.

International Workshop on Fast Neutron Detectors

University of Cape Town, South Africa

April 3 - 6, 2006 


\section{Introduction}

The construction of a new high intensity heavy ion accelerator facility demands beforehand a careful layout of its shielding design. Beam parameters like $1 \cdot 10^{12}$ uranium ions per second at an energy of $1.5 \mathrm{GeV} / \mathrm{u}$ like in the case of FAIR[1] demands precise calculations of the shielding thickness as they are of several meters of concrete an thus represent a large amount of the total costs. Up to date there are not many Monte-Carlo transport codes that can handle primary heavy ions. One method to circumvent this problem is the use of measured neutron yields as source terms for Monte-Carlo transport calculations. The neutrons represent by far the largest part of radiation of a typical heavy ion facility after some meters of concrete.

Another possibility to design shielding of simple geometries is to use a line-of-sight model. The parameters needed for this simpler approach can also be extracted from double differential neutron yields (see e.g. [2]). Last but not least double differential neutron yields may deliver a first guess of doses delivered to a person while undergoing tumor irradiation with particle beams [3].

\section{Experiments and Simulations}

Three different projectile-target combinations have been used to measure the neutron yields induced by heavy ions beams. The same combinations were also taken for the corresponding simulations, see Table 1.

Table 1: Projectile-target combinations which have been chosen for the experiments and the simulations.

\begin{tabular}{|c|c|c|c|c|}
\hline projectile & primary energy & target & $\begin{array}{c}\text { cross-sectional } \\
\text { area (target) }\end{array}$ & $\begin{array}{c}\text { thickness } \\
\text { (target) }\end{array}$ \\
\hline $\mathrm{C}-12$ & $400 \mathrm{MeV} / \mathrm{u}$ & graphite & $10 \times 10 \mathrm{~cm}^{2}$ & $20 \mathrm{~cm}$ \\
\hline $\mathrm{C}-12$ & $1000 \mathrm{MeV} / \mathrm{u}$ & iron & $10 \times 10 \mathrm{~cm}^{2}$ & $20 \mathrm{~cm}$ \\
\hline $\mathrm{U}-238$ & $1000 \mathrm{MeV} / \mathrm{u}$ & iron & $10 \times 10 \mathrm{~cm}^{2}$ & $20 \mathrm{~cm}$ \\
\hline
\end{tabular}

All experiments were carried out at Cave B of GSI. This experimental area has an area of roughly $7 \times 20 \mathrm{~m}^{2}$ and a height of $4.8 \mathrm{~m}$. A time-of-flight method with a veto detector for charged particles was used to measure the double differential neutron yields. The flight distance was varied between 3 and $7 \mathrm{~m}$. A $\Delta$ E-E-telescope [3,4] with a hexagonally (inner diam.: 8.75 $\mathrm{cm})$ shaped stop-detector was used to detect the neutrons. Photons were discriminated via a pulse shape analysis. The energy resolution of the neutrons varied between $\sim 30 \%$ for $1 \mathrm{GeV}$ neutrons and short flight distances and $\sim 3 \%$ for $100 \mathrm{MeV}$ neutrons at maximum flight distance in forward directions. The angular resolution is given by roughly $1^{\circ}$.

Table 2 describes all the detectors used for the measurements. Details can be found in [4]. In addition the large area neutron detector LAND [5] was used to measure the neutron yields at forward angles from the uranium beam [6]. LAND is built from 200 modules, each of $0.1 \times 2 \mathrm{~m}^{2}$ cross section and $10 \mathrm{~cm}$ depth. The modules consist of alternating layers (5 mm thickness) of 
iron and organic scintillator material. A multiplicity of charged particles is produced when neutrons are hitting LAND. The modules are arranged in 10 layers, crosswise positioned in subsequent layers. The scintillation light of each module is read out from the two far front faces by phototubes delivering timing information, from which time of flight and position of incidence is derived. Various sources contribute to the overall resolution in neutron kinetic energy $T_{n}$ : the intrinsic LAND time-of-flight resolution $(\sim 1 \mathrm{~ns})$, the time spread resulting from the $10 \mathrm{~cm}$ depth of each LAND module, and the time spread in the production of neutrons arising from the $20 \mathrm{~cm}$ target thickness. Adding in quadrature, the standard deviations of these effects, the energy resolution varies from $\sim 3 \%$ at $100 \mathrm{MeV}$ neutron energy to $\sim 20 \%$ at $1 \mathrm{GeV}$ neutron energy. The values are practically independent of the measured neutron angle. The angular resolution is smaller than $0.5^{\circ}$.

Table 2: detector parameters of the $\triangle E$-E-telescope

\begin{tabular}{|c|c|c|}
\hline detector & material & thickness \\
\hline start-detector & NE102 & $1 \mathrm{~mm}$ \\
\hline veto-detector & NE102A & $9 \mathrm{~mm}$ \\
\hline stop-detector & BaF2 & $14 \mathrm{~cm}$ \\
\hline
\end{tabular}

The simulations of the double differential neutron yields were performed with the MonteCarlo transport Code FLUKA [7,8], see also www.fluka.org. FLUKA has the capability of simulating interaction and propagation in matter for large a variety of particles in wide energy ranges. Recently the transport of heavy ions including nucleus-nucleus collisions has been added employing the RQMD[9] and the DPMJET[10] models.

The target dimensions and materials were properly simulated. A virtual yield detector was employed for all angles measured in the experiment with a resolution of $1^{\circ}$. For most of the simulations scattering effects due to the walls of the experimental area were not taken into account.

\section{Results}

All measured double differential yields are shown with closed circles and black error bars. The corresponding simulated yields are shown without symbols and error bars with wide caps. The different colors in the figures 1 to 6 represent always the same angle of the neutron yield with respect to the primary beam. 


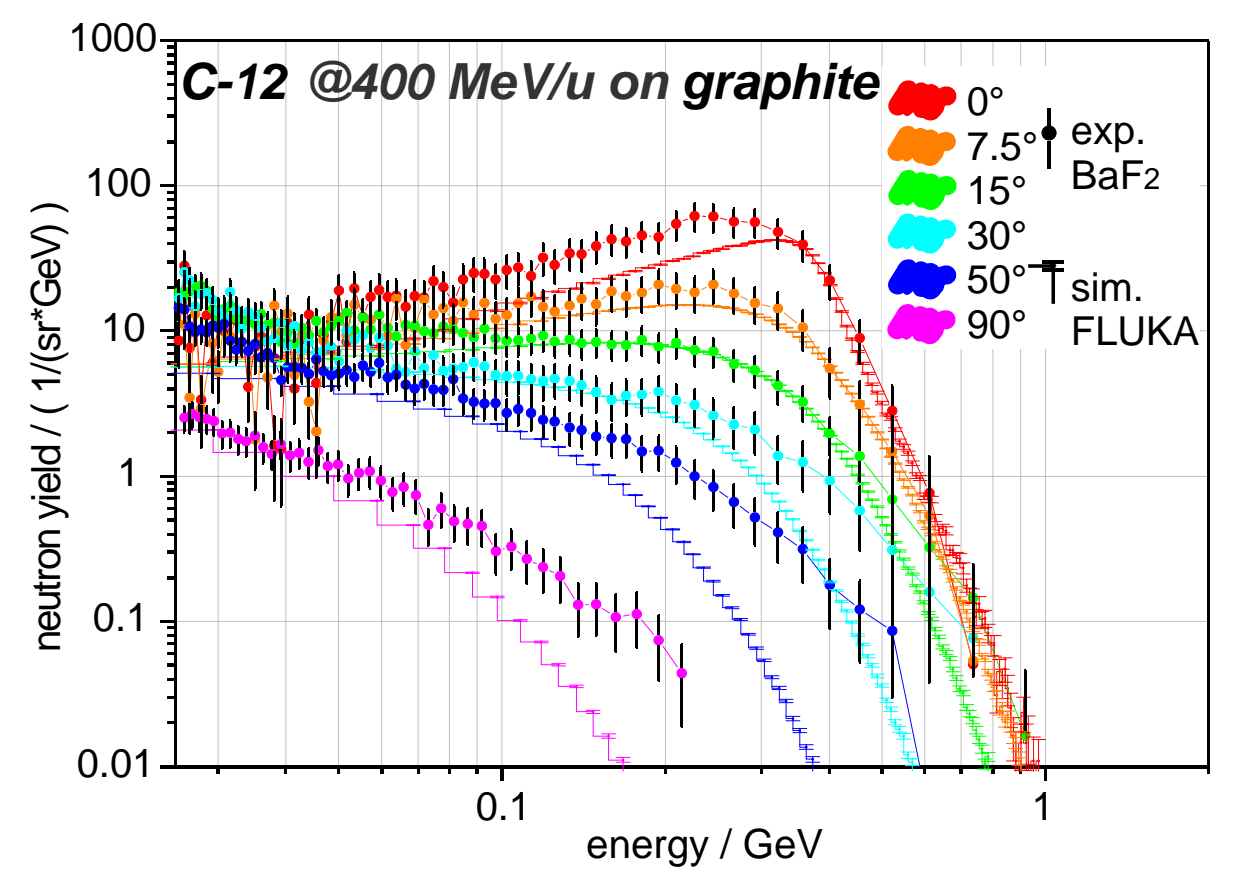

Figure 1: Double differential neutron yields induced by a $400 \mathrm{MeV/u}$ carbon beam hitting a graphite target. The black error bars at the circles represent all errors taken into account for the measurement with the $\mathrm{BaF}_{2}$-scintillator.

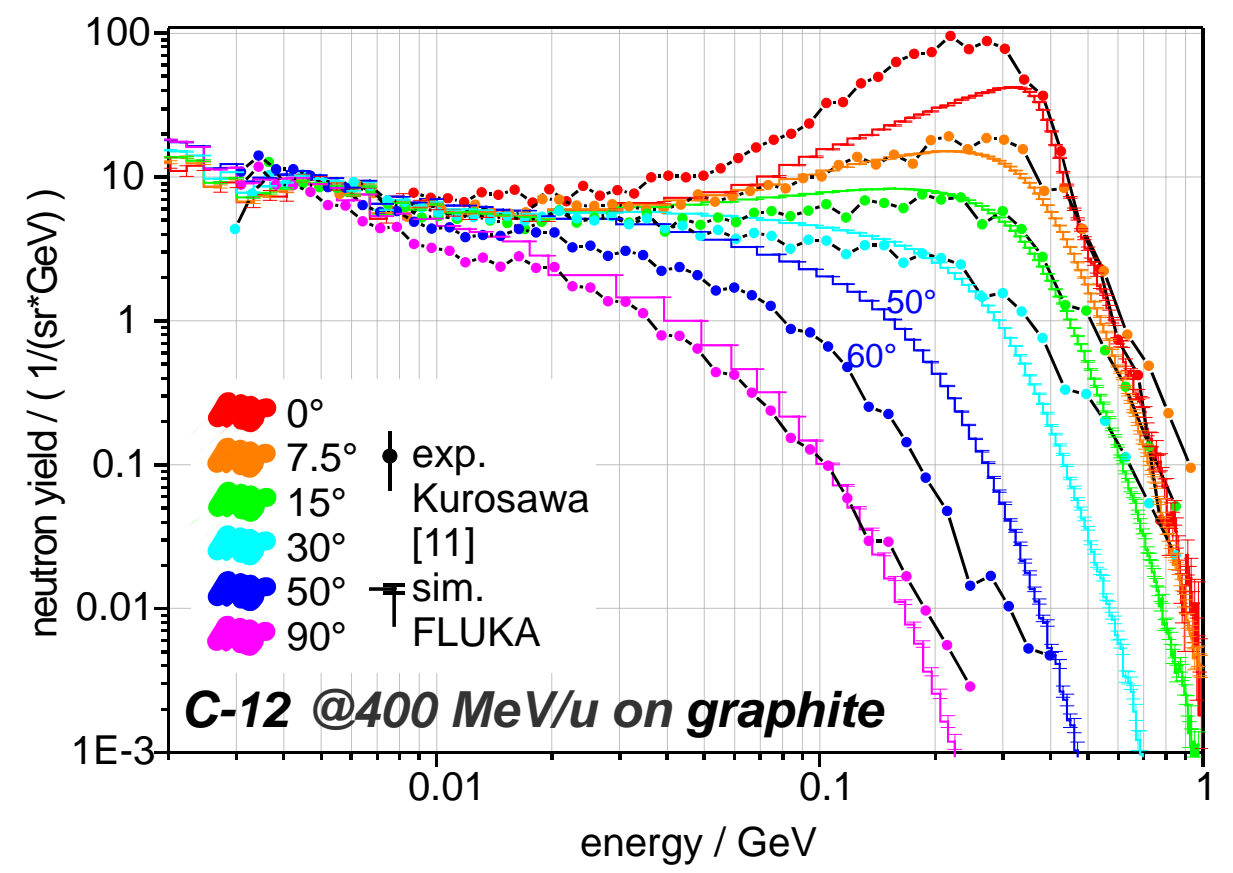

Figure2: Double differential neutron yield induced by a $400 \mathrm{MeV/u}$ carbon beam hitting a graphite target. The closed circles represent the values measured by Kurosawa et al.[11]. 
The Figures 1 and 2 show the simulated neutron yields of $400 \mathrm{MeV} / \mathrm{u}$ carbon beam on a graphite target in comparison with yields measured at GSI (Fig. 1) and yields measured by Kurosawa et al.[11] (Fig. 2). Due to the Fermi-motion of the nucleons the neutron distribution reaches energies of up to $1 \mathrm{GeV}$. The blue curves in Figure 2 cannot directly be compared as the measurements at GSI were done for an angle of $50^{\circ}$ whereas the values measured by Kurosawa represent the angle of $60^{\circ}$. Taking an error of $15 \%$ into account, as reported by Kurosawa, the overall agreement between the simulation and the experiments except for the $0^{\circ}$-direction is satisfactory.

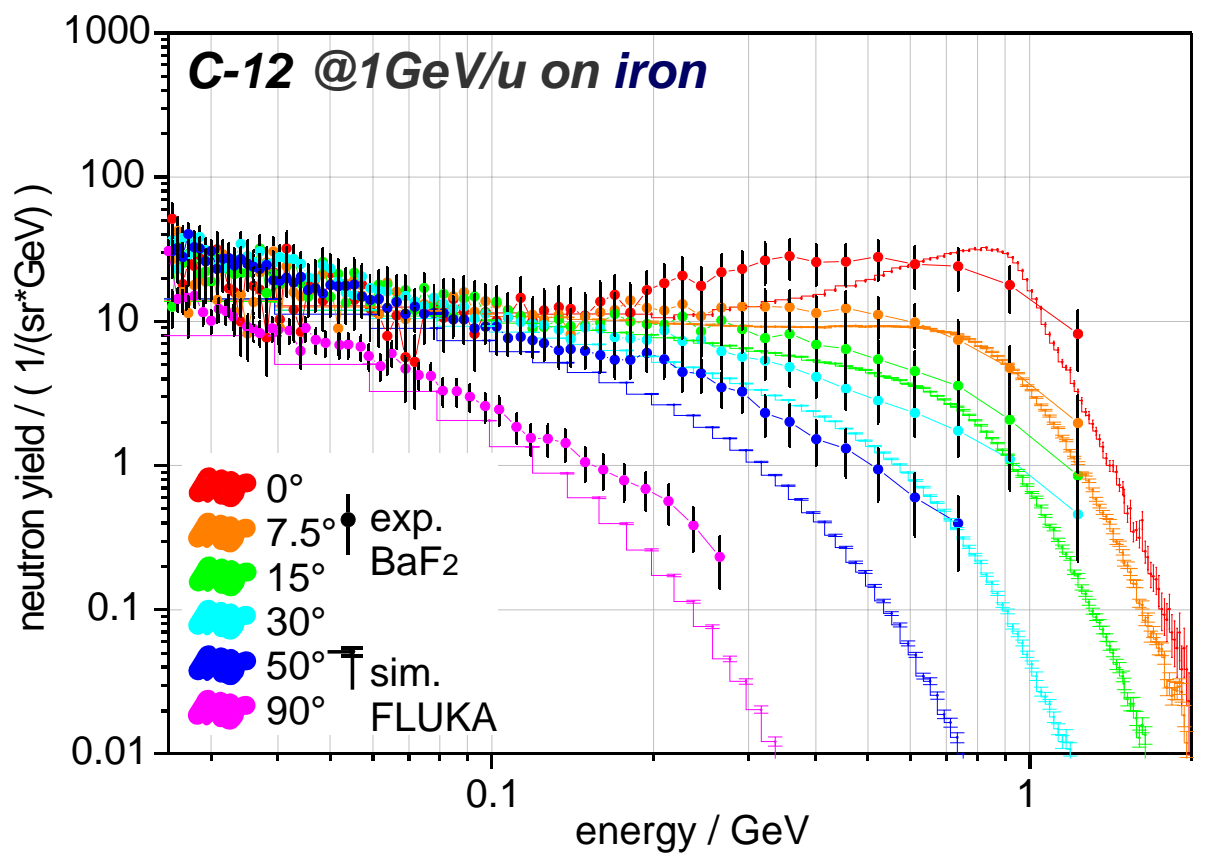

Figure 3: Double differential neutron yields induced by a $1 \mathrm{GeV/u}$ carbon beam impinging on an iron target.

Figure 3 shows the yield of a $1 \mathrm{GeV} / \mathrm{u}$ carbon beam impinging on an iron target. The yield distributions reach up to energies of more than $1.0 \mathrm{GeV}$ for the forward angles but the maximum of the neutron yields for the measured and for the simulated data is below those for $400 \mathrm{MeV} / \mathrm{u}$ carbon beam on the graphite target. Thus the maximum is broader which can be explained by a wider energy range in which nuclear collisions took place. However the simulation shows a more distinct maximum for the $0^{\circ}$-direction. Neutrons with energies of more than 1.2 MeV could not be resolved from the photons which have been discriminated. Thus the largest neutron energies could not be detected. However for the investigations of the total yield these neutrons play only a minor role due to their rapidly decreasing fluence in this energy range. 


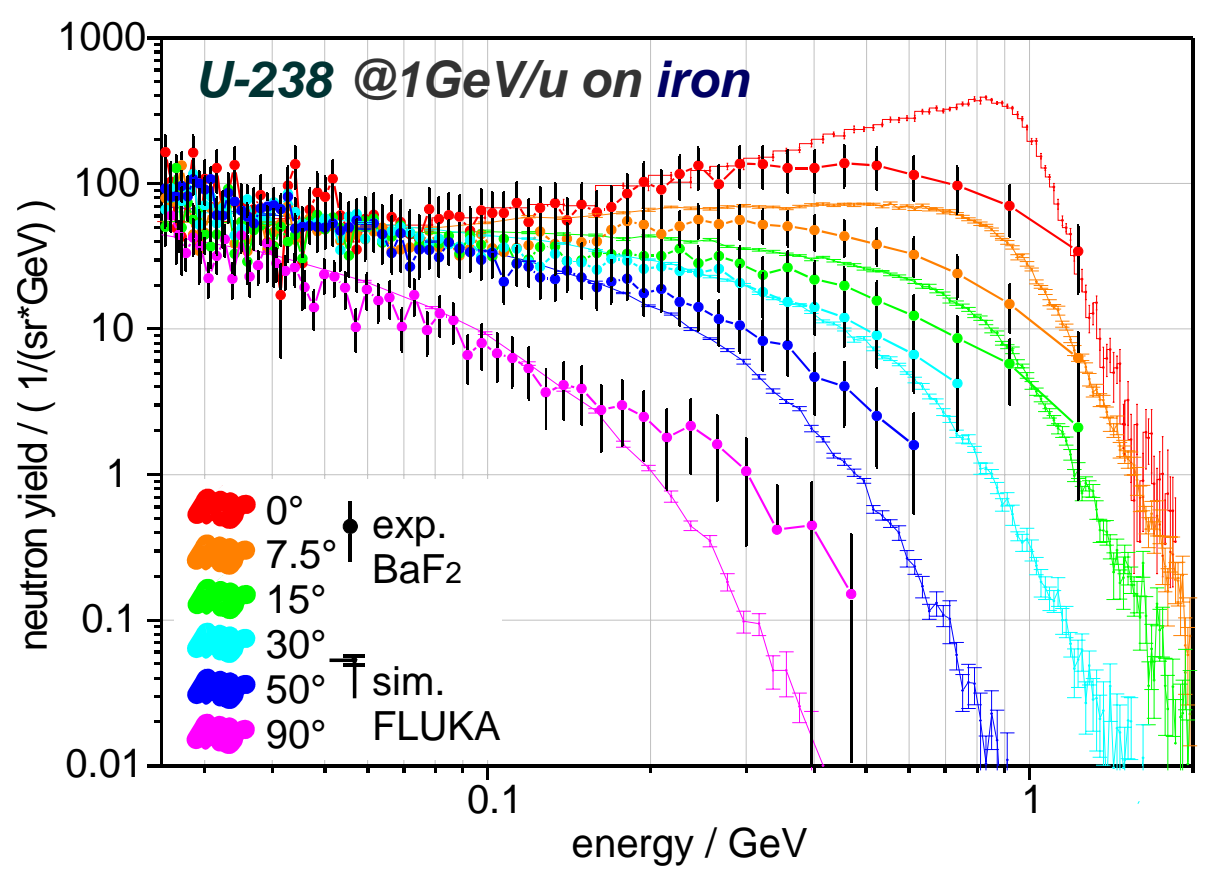

Figure 4: Double differential neutron yield induced by a $1 \mathrm{GeV/u}$ uranium beam impinging on an iron target.

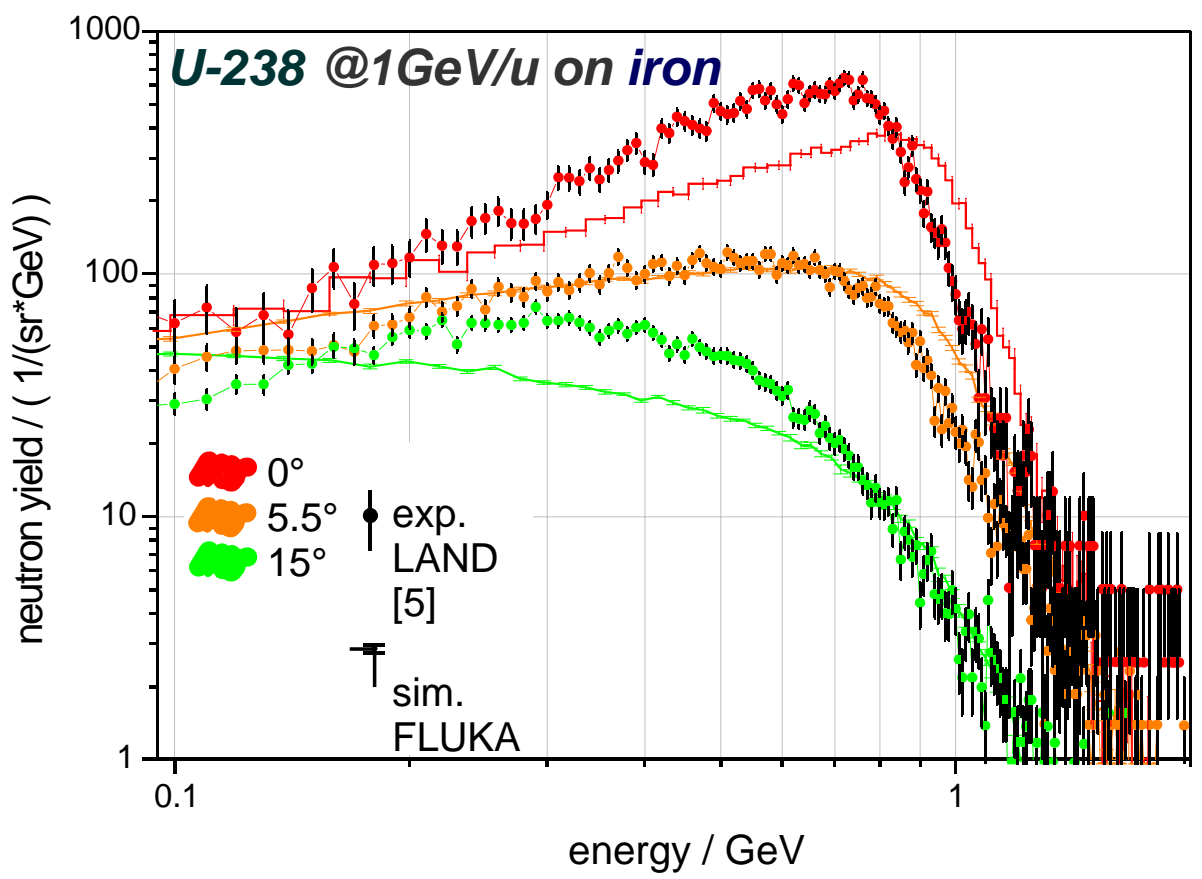

Figure 5: Double differential neutron yield induced by a $1 \mathrm{GeV} / \mathrm{u}$ uranium beam hitting an iron target. The black error bars at the circles represent the values measured with the LAND detector.

The figures 4 and 5 show neutron yields of a $1 \mathrm{GeV} / \mathrm{u}$ uranium beam impinging on an iron target. Figure 4 shows the comparison of the simulated yields with the measurements of the 
BaF2 detector whereas Figure 5 presents the comparison with the measurements performed with LAND. Considerable differences in the experimental results can be found for the $0^{\circ}$ and the $7.5^{\circ}$ -direction between the two different detectors. The simulation data overestimates the measurements with the $\mathrm{BaF}_{2}$ detector and underestimates the values delivered by LAND. The simulation underestimates the experimental yields for larger angles and high energies.

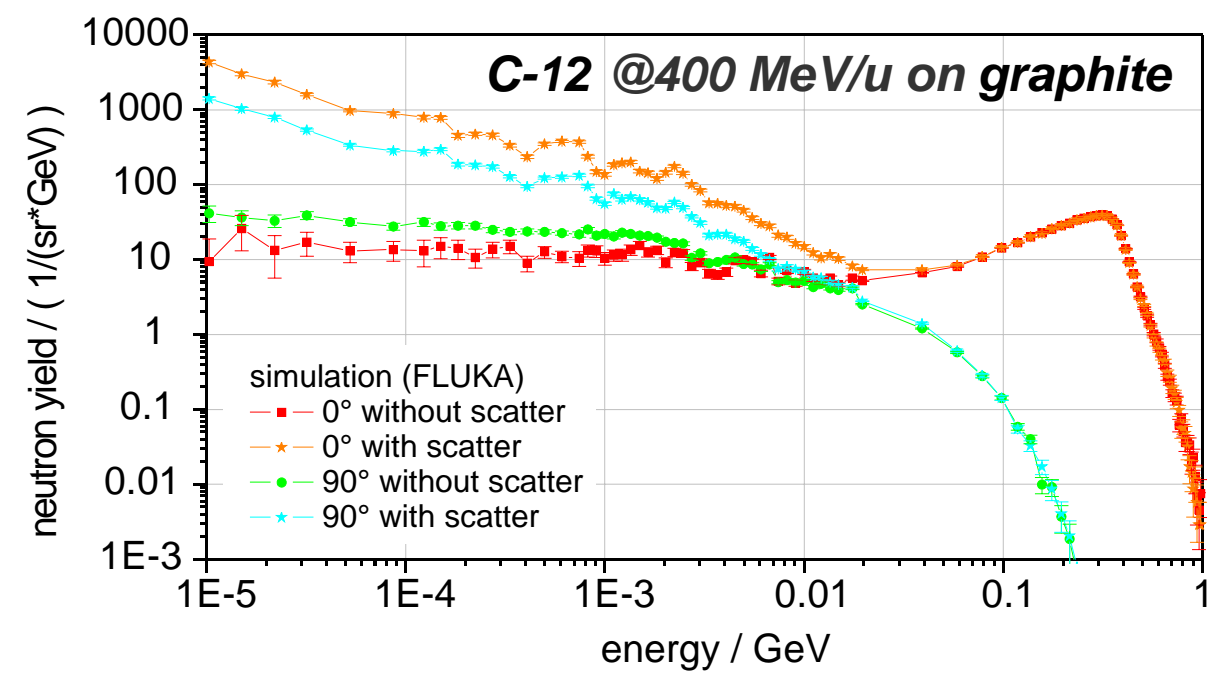

Figure 6: Simulated double differential neutron yield induced by a $400 \mathrm{MeV/u}$ carbon beam impinging on a graphite target. A rough model of Cave B including the main walls, ceiling and floor was used in order to investigate the scatter effects.

As mentioned above all simulations were performed with the only matter being the target itself. The experimental values however may contain a small amount of scattered neutrons which have hit any of the walls of the experimental area before hitting the detector. Thus another Monte-Carlo simulation was performed including a rough geometry of the caves including walls, floor and ceiling. Other parts of the facility like magnets, beam tubes, cables, electronic racks etc. were not taken into account. Figure 6 shows the neutron yields for the angles of $0^{\circ}$ and $90^{\circ}$ with and without scattering. It can be seen that above $10 \mathrm{MeV}$ there is nearly no influence of the scattering effect on the yields. However there is a large difference for the lower energetic neutrons $(<10 \mathrm{MeV})$ which is stronger pronounced in the case of the forward angle. But as the detectors used for the measurements have nearly no response for neutrons below $10 \mathrm{MeV}$ differences of simulated and measured data cannot be explained by scattering.

\section{Conclusions}

The comparison between simulated and measured double differential neutron yields shows satisfactory agreement for low-Z projectiles in the intermediate energy range. These types of 
yields can certainly be used for example to estimate doses for patients undergoing particle therapy.

For higher energetic beams and heavier projectiles like uranium investigated in the current work the deviations of experiment and simulation need to be clarified by more measurements with various projectile and target combinations. The Monte-Carlo calculation with FLUKA contains even electromagnetic interactions like electromagnetic dissociation which contributes to a small fraction in the very forward direction. Calculated yields can certainly be used for various applications like transport calculations of neutrons through thick layers of dense matter as used e.g. for shielding design of future high intensity heavy ion accelerator facilities.

\section{References}

[1] W.F. Henning, Plans for the future facility at GSI, in: J.M. D'Auria, J. Thomson, M. Comyn (Ed.), Proceedings of the 14th conference on electromagnetic isotope separators and techniques related to their applications, Victoria, BC, Canada, 6-10 May 2002, Nucl. Instr. and Meth. B 204 (2003) 725.

[2] S. Agosteo, G. Fehrenbacher, M. Silari, Attenuation curves in concrete of neutrons from $1 \mathrm{GeV} / \mathrm{u} \mathrm{C}$ and $\mathrm{U}$ ions on a Fe target for the shielding design of RIB In-Flight facilities, Nucl. Instr. and Meth. B 226 (2004) 231.

[3] K. Gunzert-Marx, Nachweis leichter Fragmente aus Schwerionenreaktionen mit einem BaF2-Teleskop-Detektor (Messungen im Rahmen des Tumortherapieprojekts der GSI), PHDwork (in German), Technical University Darmstadt, January 2004.

[4] K. Gunzert-Marx, D. Schardt, R.S. Simon, F. Gutermuth, T. Radon, V. Dangendorf and R. Nolte, Response of a BaF2 scintillation detector to quasi-monoenergetic fast neutrons in the range of 45 to $198 \mathrm{MeV}$, Nuclear Instruments and Methods A 536 (2004) 146.

[5] Th. Blaich et al., A large area detector for high-energy neutrons, Nuclear Instruments Methods A 314 (1992) 136.

[6] O. Yordanov, K. Gunzert-Marx, P. Adrich, T. Aumann, K. Boretzky, H. Emling, G. Fehrenbacher, F. Gutermuth, H. Iwase, H. Johannson, K. L. Jones, A. Kovalov, T. Radon, D. Schardt, Neutron yields from $1 \mathrm{GeV} /$ nucleon U-238 ion beams on Fe target, Nuclear Instruments and Methods B 240 (2005) 863.

[7] A. Fassò, A. Ferrari and P.R. Sala, Electron-photon transport in FLUKA: status, in: A. Kling, F. Barao, M. Nakagawa, L. Tavora, P. Vaz (Eds.), Proceedings of the Monte Carlo 2000 Conference, Lisbon, 23-26 October 2000, Springer-Verlag Berlin (2001) 159.

[8] A. Fassò, A. Ferrari, J. Ranft and P.R. Sala, FLUKA: status and prospective for hadronic applications, in: A. Kling, F. Barao, M. Nakagawa, L. Tavora, P. Vaz (Eds.), Proceedings of the Monte Carlo 2000 Conference, Lisbon, 23-26 October 2000, SpringerVerlag Berlin (2001) 955.

[9] H. Sorge, Flavor production in $\mathrm{Pb}(160 \mathrm{~A} \mathrm{GeV})$ on $\mathrm{Pb}$ collisions: Effect of color ropes and hadronic rescattering, Physical Review C 52 (1995) 3291.

[10] J. Ranft, New features in DPMJET version II.5 Phys. Rev. D51 (1995) 64. 
[11] T. Kurosawa et al., Measurements of secondary neutrons produced from thick targets bombarded by high-energy helium and carbon ions, Nucl. Sci. and Eng. 132 (1999) 30. 